See discussions, stats, and author profiles for this publication at: https://www.researchgate.net/publication/327103566

\title{
TWO-DIMENSIONAL TRANSIENT HEAT TRANSFER AND OPTICAL ANALYSIS OF A SOLAR RECEIVER
}

Conference Paper · August 2018

CITATIONS

0

3 authors, including:

6. Nesrin Ozalp University of Minnesota Duluth

90 PUBLICATIONS 714 CitATIONS

SEE PROFILE
READS

7

Cédric Ophoff

KU Leuven

6 PUBLICATIONS 11 CITATIONS

SEE PROFILE

Some of the authors of this publication are also working on these related projects:

Effect of a Variable Aperture Mechanism on the Dynamics of Solar Reactors View project

Solar Thermal Hydrogen Production View project 


\title{
TWO-DIMENSIONAL TRANSIENT HEAT TRANSFER AND OPTICAL ANALYSIS OF A SOLAR RECEIVER
}

\author{
Mostafa Abuseada ${ }^{1}$, Nesrin Ozalp ${ }^{1 *}$, Cedric Ophoff ${ }^{2}$ \\ ${ }^{1}$ University of Minnesota Duluth, Duluth, MN 55812, USA \\ ${ }^{2} \mathrm{KU}$ Leuven, Leuven 3001, Belgium
}

\begin{abstract}
Transient nature of solar radiation creates challenges in operating solar reactors requiring semi-constant cavity temperatures. A promising approach to overcome this problem is to implement a variable aperture. This paper presents a heat transfer model of a solar reactor featuring a variable aperture. The model is an in-house developed code comprising transient two-dimensional heat transfer analysis. The model is coupled with an optical analysis based on the Monte Carlo Ray Tracing (MCRT) or the Radiosity Net Exchange (RNE) method to simulate radiation within the receiver. The model is also coupled with a fluid dynamics analysis based on implementing a staggered grid system and the SIMPLE algorithm to obtain the velocity field of the fluid flow. Validation of the model was made through experimental results obtained using a $7 \mathrm{~kW}$ high flux solar simulator with an input current of $155 \mathrm{~A}$ and a fully opened aperture at $60 \mathrm{~mm}$ radius. Results showed satisfactory accuracies given an experimental maximum uncertainty of $\pm 8.6^{\circ} \mathrm{C}$. However, the MCRT method provided more accurate results. The difference between the experimental and the model's steady state temperature values had an average of $6.3^{\circ} \mathrm{C}$ and $11.8^{\circ} \mathrm{C}$ for the MCRT and RNE methods, respectively. Based on the temperature distribution results, it was noted that the RNE method fails to predict accurate radiation distribution where two surfaces meet at perpendicular angles. Finally, the in-house code was used to determine an optimum aperture radius per highest possible exhaust temperature value which was $52.5 \mathrm{~mm}$ for the operating conditions outlined above.
\end{abstract}

KEY WORDS: Solar energy, Radiation, Computational methods, Optical analysis, Solar receiver, Variable aperture

\section{INTRODUCTION}

Solar radiation is concentrated through a heliostat field to provide receivers with high temperature heat. A solar receiver is a cavity that aims for maximizing the captured radiation, while minimizing the re-radiation lost. The high temperature heat can then be used in various chemical and non-chemical process applications including production of electricity, metals, fuels, and many other products [1-3]. However, the inconsistency of intercepted solar irradiance on the Earth's surface creates a challenge in operating solar receivers. This is due to the requirement of semi-constant temperatures within the solar reactor's cavity in order to maximize the process efficiency.

There have been many studies on the challenge of achieving semi-constant temperatures within a solar receiver. These efforts have created remarkable designs for solar reactors housing various thermochemical processes [2-4]. However, these reactors have fixed apertures that do not compensate for the fluctuation of the solar irradiance. The optimum aperture size is identified through a compromise between maximum radiation captured and minimum re-radiation lost as illustrated by Steinfeld and Schubnell [5]. Many others approached the challenge of maintaining constant temperatures by adjusting the flowrate of the feedstock [6-7]. However, this method is not preferred in processes where a certain flow pattern needs to be preserved. Therefore, a variable aperture is a promising technique to accommodate for the inconsistency of solar irradiance.

*Corresponding Author: nozalp@d.umn.edu 
To effectively control the variable aperture, the solar receiver has to be modelled comprehensively. Therefore, an in-house code comprising transient two-dimensional heat transfer model across the solid and fluid phases was developed. The model starts by simulating the incident radiation from the source and re-radiation within the solar receiver. This can be established through several optical methods; such as the Radiosity Net Exchange (RNE) and Monte Carlo Ray Tracing (MCRT) methods. The MCRT method has been the preferred method over the simpler RNE method for simulating radiation $[2,8]$. In present paper, both methods were incorporated in the model and their results were compared. For the heat transfer part of the model, a fluid dynamics analysis needs to be incorporated to accurately model the fluid phase. There are many different methods, based on finite volume analysis that can be implemented. These methods can be divided into two categories: staggered and co-located grid-system based methods. Co-located grid methods require the implementation of projection methods to avoid specious pressure oscillations. Some of these methods include the pressure-weighed interpolation, Rhie-Chow interpolation, and artificial compressibility method [9-11]. On the other hand, staggered grid methods include the Semi-Implicit Method for Pressure-Linked Equations (SIMPLE) and SIMPLE Revised algorithms. In these methods, the discretised governing equations would contain differences of adjacent components rather than alternating components as in the co-located methods. Therefore, the SIMPLE algorithm has been incorporated in the model presented due to its implementation of the staggered grid system and lower computational effort over the SIMPLER method [12].

\section{EXPERIMENTAL SETUP}

\subsection{System Description and Overall Setup}

The system presented here consists of a cavity type solar receiver with a variable aperture along with a high flux solar simulator as the radiative source. The radiative source used consists of a $7 \mathrm{~kW}$ Xenon arc high discharge lamp that can deliver varied power levels depending on the current supplied. The fluid flow through the solar receiver was Nitrogen at 10 standard liters per minute (SLPM) for this experimental testing stage. The system is shown in Fig. 1a with the aperture removed to allow for a better visualization of the setup.

The solar receiver consists of a hollow cylinder with a front plate and a back plate bolted to it forming the cavity of the receiver. The cavity is secured by a quartz window, and the variable aperture mechanism then mounts on the front plate. Details of the variable aperture mechanisms for this setup can be found elsewhere [13]. The cylinder cavity has a length of $200 \mathrm{~mm}$ and an inner radius of $60 \mathrm{~mm}$. The thicknesses of the cylinder, front plate, and back plate are 15,25 , and $15 \mathrm{~mm}$, respectively. The solar receiver has three inlet ports for the fluid flow, 10 ports to measure the temperature distribution across the cavity, and a single port to measure the exhaust temperature. An illustrated CAD model is shown in Fig 1b. The solar receiver is wrapped by $40 \mathrm{~mm}$ thick insulation material.
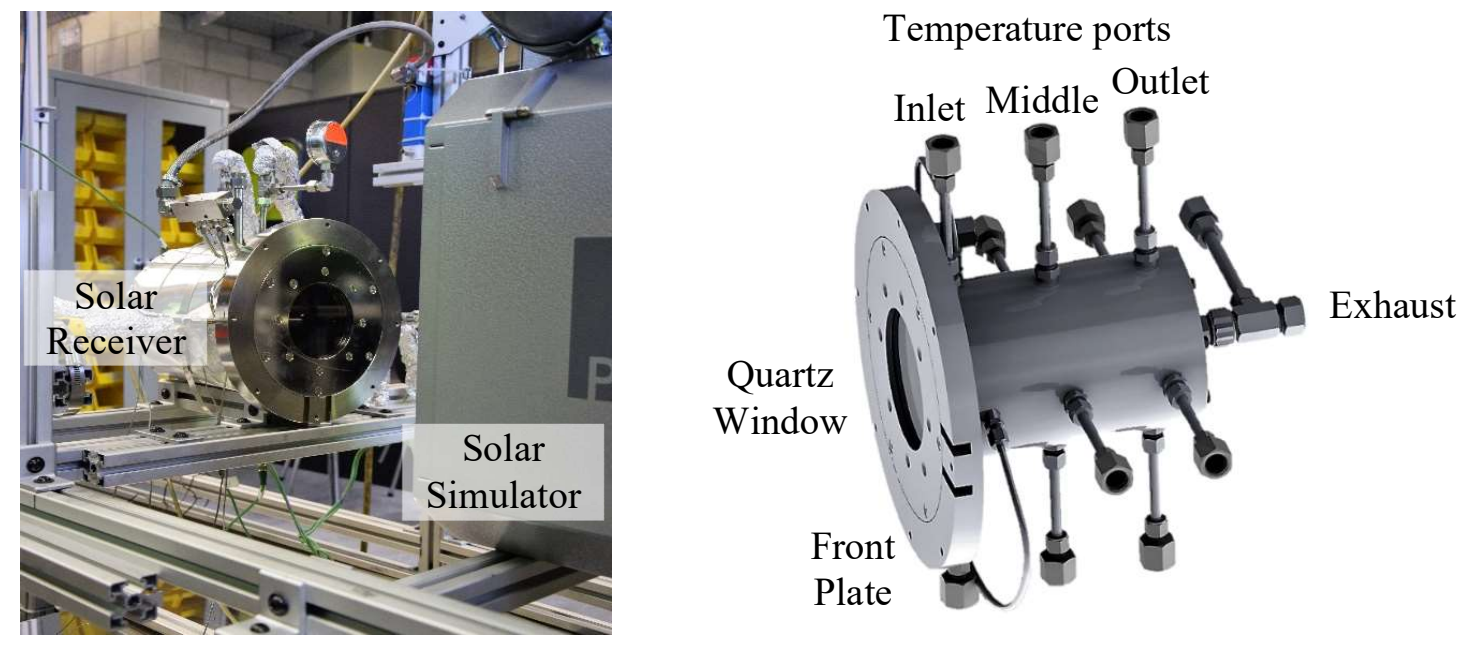

Fig. 1 (a) Experimental setup of the receiver and radiative source, and (b) CAD model of the receiver tested. 


\subsection{Optical Characterization of the Solar Simulator}

It is essential to quantify the power input from the solar simulator to accurately model the heat transfer across the solar receiver. Therefore, the radiative power incident at the focal plane was characterized experimentally through a heat flux measurement. This was performed through an indirect flux mapping technique that incorporates the use of a heat flux gage, two Lambertian targets, and a complementary metal oxide semiconductor (CMOS) camera along with optical filters. This technique is the most commonly used as it provides a high data acquisition and spatial resolution [14]. The experimental setup and method used for the characterization is further explained and illustrated elsewhere [15]. The experimentally conducted optical characterization was based on a supply current of $155 \mathrm{~A}$ to the solar simulator which provided a total of $903 \mathrm{~W}$ on an $8 \times 8 \mathrm{~cm}^{2}$ target with a maximum flux of $3159 \mathrm{~kW} / \mathrm{m}^{2}$ at the center. Fig. 1a shows the measured three-dimensional heat flux distribution at the focal plane on an $8 \times 8 \mathrm{~cm}^{2}$ target along with Fig. $2 \mathrm{~b}$ that shows a magnified flux contour map for an area of $2 \times 2 \mathrm{~cm}^{2}$.

\section{NUMERICAL MODEL}

The full optical and heat transfer numerical model presented here combines various methods to accurately simulate the heat transfer within the solar receiver. The model begins with an optical analysis where the MCRT method was implemented to simulate the power distribution from the energy source to the receiver. Then, either the MCRT method or the RNE method was incorporated to simulate the distribution of the radiation emitted from the enclosure. The optical analysis was then coupled with a set of governing equations that describe the system studied. These governing equations include the mass, momentum, and energy conservations, where a staggered grid and the SIMPLE algorithm were implemented to produce velocity-pressure coupling equations.

It is vital to begin by stating the assumptions that the model incorporates. For simplicity, the solar receiver was treated as a two-dimensional model due to symmetry with all its walls being diffuse gray surfaces. The surface properties were assumed to be independent of temperature, and the fluid flow was treated as a non-participating medium. Finally, the fluid was assumed to be incompressible due to the Mach number being less than 0.3 . With regards to the boundary conditions, the velocity was defined at the inlet to maintain a constant flow of 10 SLPM. The ambient temperature surrounding the receiver was assumed to remain constant at a room temperature of $20^{\circ} \mathrm{C}$. Although extended run of the solar simulator and receiver would potentially increase this temperature slightly, precautions were taken to ensure that it remained constant. The insulation thickness value, run time, and frequency of experiments allowed for the use of the laboratory's regular ventilation system to maintain the room temperature without affecting the experimental run.

\subsection{Optical Analysis}

Monte Carlo Ray Tracing method. The MCRT is a statistically based method that functions by emerging randomly determined paths of a large number of rays and tracing them as they interact with the surfaces of an enclosure.
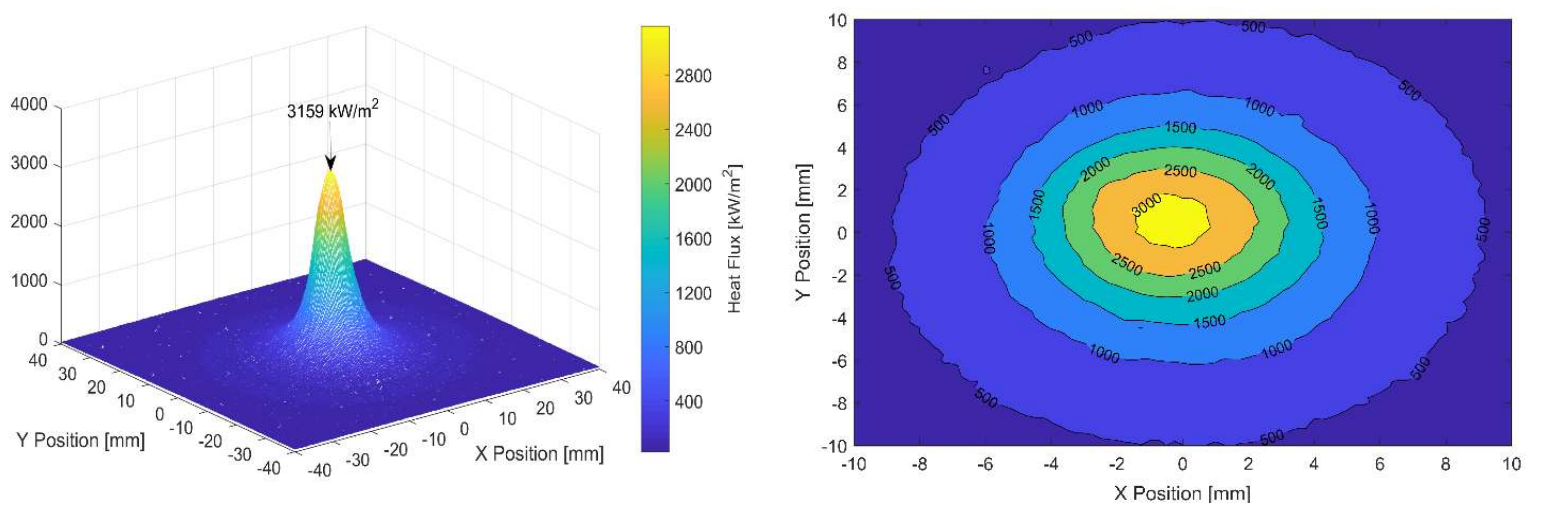

Fig. 2 Heat flux distribution at input current of $155 \mathrm{~A}$ (a) $8 \times 8 \mathrm{~cm}^{2} 3 \mathrm{D}$ distribution, and (b) $2 \times 2 \mathrm{~cm}^{2}$ contour map. 
This method incorporates the use of a quantity called the distribution/exchange factor, $D_{i j}$. This factor is defined as the fraction of total emitted radiation by a surface $i$ that is absorbed by another surface $j$ due to direct radiation and all possible reflections [16].

In modeling a radiative source or surface $i$, the quantity of radiation is divided equally into a large number, $N_{i}$, of discrete energy bundles. The value of $N_{i}$ depends on the required accuracy and computing time. However, it is generally recommended that $N_{i}$ be at least $10,000 n$, where $n$ is the total number of surface elements [16]. Either way, a convergence analysis is performed to ensure that the distribution factor converges with an acceptable accuracy. For the model presented here, 20,000n energy bundles were emitted from each radiative element where this value was more than sufficient based on the convergence analysis and comparison with other models $[2,8]$.

When tracing each energy bundle, the point of emission from the surface element is calculated randomly through the use of polar coordinates, as seen in equation (1).

$$
(r, \varphi)=\left(R_{i} \sqrt{\mathcal{R}_{s}}, 2 \pi \mathcal{R}_{s}\right)
$$

Where $\mathcal{R}_{s}$ is a randomly selected number between zero and unity in a random set $s$. A different set of random numbers should be used for every different parameter of the MCRT method. The direction of emission of the energy bundle in polar coordinates is then determined through equation (2).

$$
(\theta, \phi)=\left(\sin ^{-1} \sqrt{\mathcal{R}_{s}}, 2 \pi \mathcal{R}_{s}\right)
$$

Once the position and direction of an emission are determined, the energy bundle is traced till it intersects with an enclosure element. Then, the point of intersection is determined along with whether the bundle is absorbed or reflected through a probabilistic approach. If it is absorbed, the bundle will be recorded in a counter, $N_{i j}$, where $i$ is the emitting surface element and $j$ is the absorbing surface. However, if it reflected, the direction of reflection needs to be determined through equation (2). This procedure continues till all $N_{i}$ bundles have been emitted and recorded where finally the distribution factor can be determined through equation (3).

$$
D_{i j}=\frac{N_{i j}}{N_{i}}
$$

Radiosity Net Exchange method. The RNE method is based on a purely geometric factor called the configuration/view factor defining the geometrical relationship between any two surface elements. The direct view factor, $F_{i j}$, then represents the fraction of radiation leaving surface element $i$ that arrives at surface element $j$ by direct radiation only without taking into consideration any intermediate reflections [16]. The direct view factor may then be used alongside the view factor summation and reciprocity relations in order to make it an indirect method. Thus, taking into consideration any intermediate reflections too. Equation (4) shows the summation and reciprocity relations used.

$$
\sum_{j=1}^{n} F_{i j}=1.0 \quad \text { and } \quad A_{i} F_{i j}=A_{j} F_{j i} \quad 1 \leq i \leq n
$$

There are many useful view factors that have been calculated and published [16-17]. However, it is important to validate these factors and adjust them accordingly through the conservation of energy and reciprocity rules of the view factors shown in equation (4). The view factors used for this optical model were obtained from [17] and adjusted as necessary. After all the view factors have been calculated, equation (5) and equation (6) were then used to calculate the radiation leaving a surface (radiosity) and radiation absorbed by a surface element, respectively.

$$
q_{r a d, i}=q_{e m i t, i}+\left(1-\alpha_{i}\right) \sum_{j=1}^{n} q_{r a d, j} F_{j i}=A_{i} \varepsilon_{i} \sigma T_{i}^{4}+\rho \sum_{j=1}^{n} q_{r a d, j} F_{j i} \quad 1 \leq i \leq n
$$




$$
q_{a b s, i}=\alpha_{i} \sum_{j=1}^{n} q_{r a d, j} F_{j i} \quad 1 \leq i \leq n
$$

\subsection{Fluid Dynamics Analysis}

Considering the previously stated assumptions of the model, equations (7), (8), and (9) are obtained. These represent the conservation laws of mass, $z$-momentum, and $r$-momentum, respectively. These laws form the governing equations representing the fluid dynamics, where they should always be preserved.

$$
\begin{gathered}
\rho\left[\frac{1}{r} \frac{\partial\left(r u_{r}\right)}{\partial r}+\frac{\partial\left(u_{z}\right)}{\partial z}\right]=0 \\
\rho\left(\frac{\partial u_{z}}{\partial t}+u_{r} \frac{\partial u_{z}}{\partial r}+u_{z} \frac{\partial u_{z}}{\partial z}\right)=-\frac{\partial p}{\partial z}+\mu\left[\frac{1}{r} \frac{\partial}{\partial r}\left(r \frac{\partial u_{z}}{\partial r}\right)+\frac{\partial^{2} u_{z}}{\partial z^{2}}\right] \\
\rho\left(\frac{\partial u_{r}}{\partial t}+u_{r} \frac{\partial u_{r}}{\partial r}+u_{z} \frac{\partial u_{r}}{\partial z}\right)=-\frac{\partial p}{\partial r}+\mu\left[\frac{1}{r} \frac{\partial}{\partial r}\left(r \frac{\partial u_{r}}{\partial r}\right)+\frac{\partial^{2} u_{r}}{\partial z^{2}}\right]
\end{gathered}
$$

In order to solve for the fluid dynamics, the velocity and pressure fields must be known or calculated. By inspecting equations (7), (8), and (9), there is no direct equation that can be used to solve for the pressure field. However, equation (7) indirectly provides a method of calculating the pressure field which is performed through the SIMPLE algorithm. The algorithm starts with initial conditions for the velocity and the pressure fields. The velocity field is then solved for using equations (8) and (9) with the initial guess of the pressure field. However, these fields do not yet conserve mass. Therefore, the algorithm is used to construct a pressure correction equation derived from equation (7) to calculate the corrected pressure and velocity fields [12].

\subsection{Heat Transfer Analysis}

By having the incident radiation and re-radiation simulated as well as the fluid velocity fields calculated, the solar receiver can now be accurately modeled to provide its temperature distribution. This is achieved through the main governing equation which is constructed through the conservation of energy and couples all the previous analyses together. Incorporating the assumptions of the model in addition to neglecting the viscous dissipation due to its insignificance for Nitrogen [18], equation (10) is obtained as the main governing equation.

$$
\rho c_{p}\left(\frac{\partial T}{\partial t}+u_{r} \frac{\partial T}{\partial r}+u_{z} \frac{\partial T}{\partial z}\right)=k\left[\frac{1}{r} \frac{\partial}{\partial r}\left(r \frac{\partial T}{\partial r}\right)+\frac{\partial^{2} T}{\partial z^{2}}\right]+S
$$

Inspecting equation (10), the fluid dynamics analysis is coupled through the velocity components, while the optical analysis is coupled through the source term, $S$. This term defines the radiation absorbed as well as that emitted from an element, which is only considered for the surface elements of the solar receiver. Through this definition, equation (11) states the source term for a surface element.

$$
S_{i}=\frac{q_{a b s, i}-q_{e m i t, i}}{V_{i}}=\frac{\left(\alpha_{i} \sum_{j=1}^{n} q_{r a d, j} F_{j i}-A_{i} \varepsilon_{i} \sigma T_{i}^{4}\right)}{2 \pi R_{i} \Delta r \Delta z}=\frac{\left(\sum_{j=1}^{n} A_{j} \varepsilon_{j} \sigma T_{j}^{4} D_{j i}-A_{i} \varepsilon_{i} \sigma T_{i}^{4}\right)}{2 \pi R_{i} \Delta r \Delta z}
$$

\subsection{System Discretization}

Once the governing equations were identified, the solar receiver system was discretized accordingly. Based on treating the receiver as two-dimensional, cylindrical coordinates were the best choice for a coordinate system. Hence, the finite volume elements considered were either hollow or solid cylinders. The equations were then applied on each volume, where a staggered grid system was implemented. This system located the radial and axial velocity components at two different grid points that were also different from those of the co-located pressure and temperature components. The discretized system also incorporated the use of the upwind scheme, 
where the variable associated with any convection term had the same value as that at the grid point on the upwind side of the volume. Finally, the fully implicit scheme was incorporated when solving the governing equations due to its higher accuracy and faster convergence [12].

\section{RESULTS AND DISCUSSION}

\subsection{Model Validation}

The solar receiver model was validated by experimentally obtained results for the temperature distribution across the receiver. The experimental results were based on a supplied current of $155 \mathrm{~A}$ to the solar simulator and the variable aperture was kept fully opened at a radius of $60 \mathrm{~mm}$. Fig. $1 \mathrm{~b}$ shows the different ports within the receiver that allow for measuring the temperature values at different locations. These locations are the inlet, middle, and outlet sections of the receiver. The temperature data collected at each location was then averaged out and the mean temperature values were then plotted with respect to time along with the results obtained from the model.

Experimental results were compared with the model's results for both the MCRT and RNE methods. Fig. 3a and $4 \mathrm{a}$ show the contour plots of the temperature distribution within the receiver, while Fig. $3 \mathrm{~b}$ and $4 \mathrm{~b}$ show the experimental temperatures and the model's results at the three aforementioned locations, respectively for the MCRT and RNE models. Both the MCRT and the RNE models provided results of reasonable accuracies. Inspecting Fig. 3b, the differences between the MCRT's theoretical and experimental temperatures at the steady state were $6.6,7.7$, and $4.6^{\circ} \mathrm{C}$ for the inlet, middle, and outlet sections of the cavity, respectively.
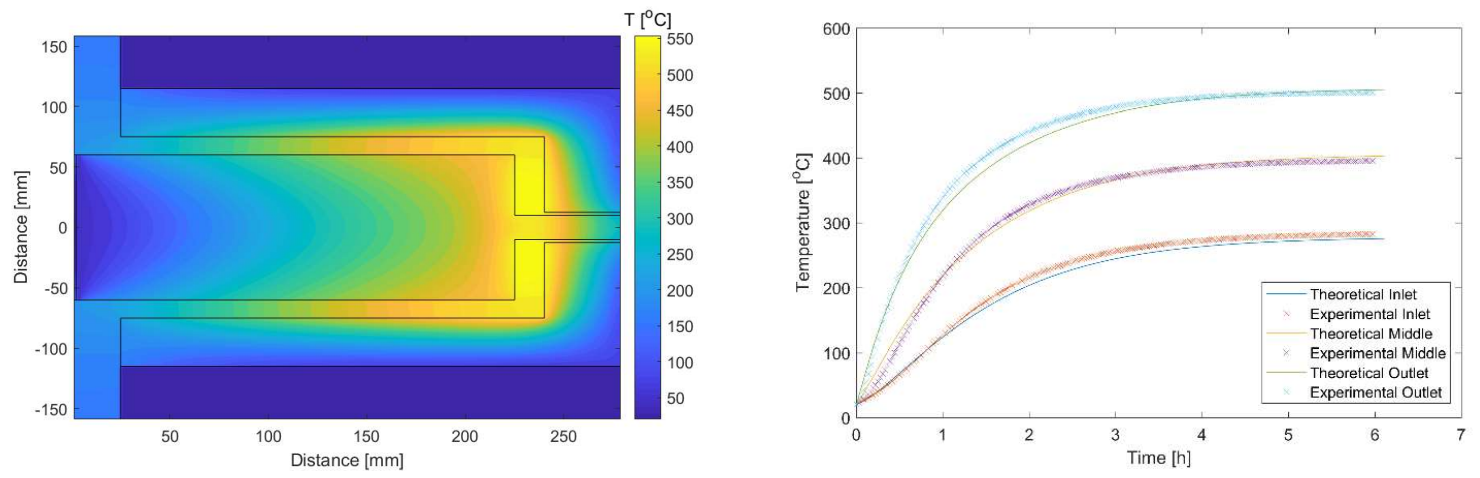

Fig. 3 MCRT model's temperature distribution (a) Contour plot of temperature distribution within cavity, and (b) Experimental and theoretical temperatures at the inlet, middle, and outlet sections of the cavity.
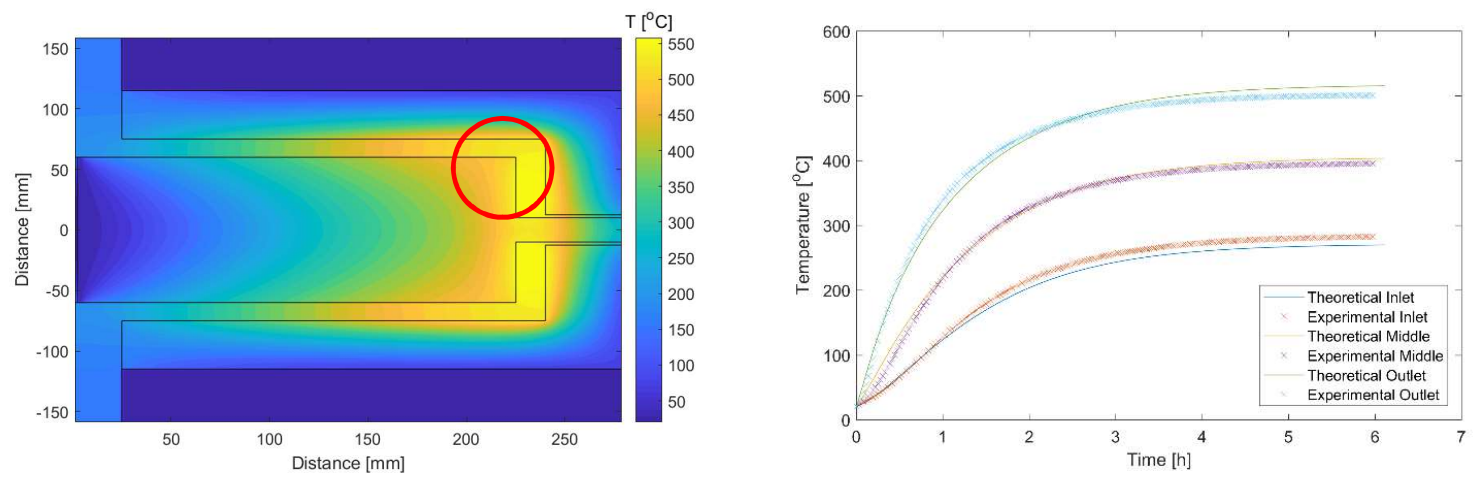

Fig. 4 RNE model's temperature distribution (a) Contour plot of temperature distribution within cavity, and (b) Experimental and theoretical temperatures at the inlet, middle, and outlet sections of the cavity. 
However, inspecting Fig. $4 \mathrm{~b}$, the RNE's steady state temperatures differences were $12.4,8.3$, and $14.8^{\circ} \mathrm{C}$, respectively. Therefore, it can be noted that the model based on the MCRT method provided more accurate results over the RNE method for the temperature distribution within the cavity, especially at the inlet and outlet sections. This is due to the reason that at these two sections, two surfaces meet at perpendicular angles (considering the aperture as an imaginary surface). Therefore, the RNE method fails to provide high accuracy results at these sections, and usually an excessively fine mesh would be necessary at these intersections. The slightly off representation of the radiation distribution is indicated in Fig. 4a, where the RNE method's contour provided higher temperature values at the outlet corner of the cavity with respect to the MCRT method.

Uncertainty analysis. To ensure that the comparison between the model and experimental results as well as any conclusions drawn are viable, an uncertainty analysis was required to be conducted. The method used for analyzing the uncertainty was acquired from the American National Standards Institute (ANSI) [19]. This method identifies uncertainty as two types; type A, which is due to the experimental setup and fluctuation in the data obtained, and type B, which is due to uncertainty of sensors (thermocouples). Type A maximum uncertainty was determined through the largest difference between the averaged experimental data and any of its constituents, which was evaluated as $\pm 3.8^{\circ} \mathrm{C}$. On the other hand, the maximum uncertainty $(0.4 \%)$ of the type $\mathrm{K}$ thermocouples used is $\pm 2.0^{\circ} \mathrm{C}$. Therefore, the maximum total uncertainty, with a $95 \%$ confidence value, is $\pm 8.6^{\circ} \mathrm{C}$, which corresponds to a maximum relative error of $3 \%$ at steady state conditions.

\subsection{Simulating Optimum Aperture Size}

The model of the solar receiver can also be used to determine the optimum radius for the variable aperture, where the fluid's exhaust temperature is maximum. This was achieved by considering the decrease in incident radiation as well as the increase in the amount of re-radiation absorbed due to the decrease in the aperture's size. The aperture's optimum radius is identified by the radius corresponding to the highest temperature value (peak) of Fig. 5 , which shows the variation of the exhaust's steady state temperature with respect to the aperture's radius. The optimum radius was determined to be $52.5 \mathrm{~mm}$ for the given operating conditions.

\section{CONCLUSIONS}

In this paper, a two-dimensional transient optical and heat transfer analysis of a solar receiver with a variable aperture was presented. The in-house code developed coupled the MCRT and RNE optical models, in addition to the SIMPLE algorithm, of the heat transfer model of the receiver. Through comparison of the model results and experimentally obtained data, it was concluded that the MCRT method provides more accurate results over the RNE method. This was especially true at sections where two surfaces meet at a perpendicular angle. Both models had a difference in steady state temperatures of $2.1 \%$ at the inlet and outlet sections of the cavity, while only $0.2 \%$ at the middle section. The optimum aperture's radius, for the experimental conditions was

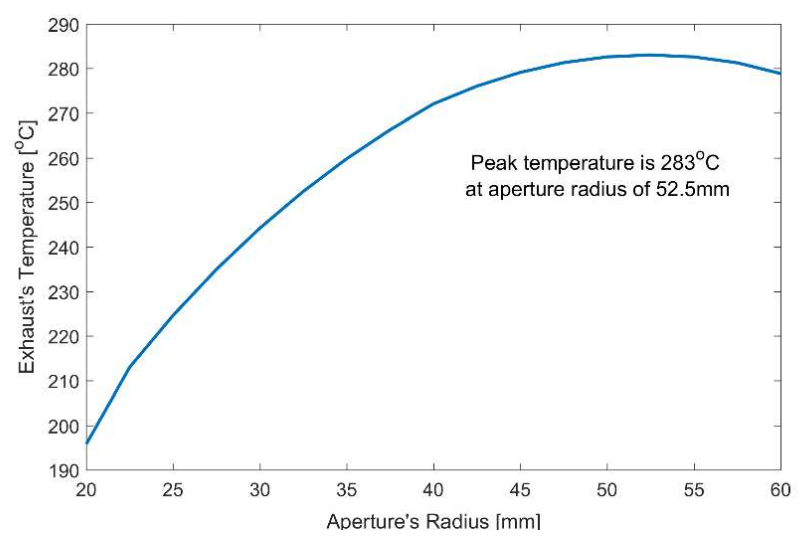

Fig. 5 Relationship between the exhaust temperature and aperture radius. 
IHTC16-24296

determined to be $52.5 \mathrm{~mm}$ through measuring the exhaust temperature for different aperture radii. Future work will include further improvement of the in-house code in addition to further examination of effects of the quartz window since it has substantial effects especially at different radiation wavelength values [3].

\title{
ACKNOWLEDGMENT
}

The experimental part of this research has been funded by Impulse Fund project \# IMP/14/049 of KU Leuven.

\section{NOMENCLATURE}

\begin{tabular}{lll}
\multicolumn{2}{l}{ Notations } \\
$S$ & energy source term & $\left(\mathrm{W} / \mathrm{m}^{3}\right)$ \\
$D$ & distribution factor & $(-)$ \\
$\mathcal{R}$ & random number & $(-)$ \\
$F$ & view factor & $(-)$ \\
$\Delta$ & increment & $(-)$
\end{tabular}

\author{
Subscripts \\ abs absorbed \\ emit emitted \\ rad radiosity \\ $\mathrm{n} \quad$ number of surface elements
}

\section{REFERENCES}

[1] Baniasadi, E., "Concurrent hydrogen and water production from brine water based on solar spectrum splitting: process design and thermoeconomic analysis," Renewable Energy, 102, pp. 50-64, (2017).

[2] Kraupl, S. and Steinfeld, A., "Monte Carlo radiative transfer modeling of a solar chemical reactor for the co-production of Zinc and Syngas," Journal of Solar Energy Engineering, 127, pp. 102-108, (2005).

[3] Hirsch, D., Hydrogen Production by the Solar Thermal Decomposition of Natural Gas using a Vortex-Flow Solar Reactor, Ph.D. Thesis, ETH Zurich, (2003).

[4] Dahl, J.K., Buechler, K.J., Weimer, A.W., Lewandowski, A., and Bingham, C., "Solar-thermal dissociation of methane in a fluid-wall aerosol flow reactor," International Journal of Hydrogen Energy, 29, pp. 725-736, (2004).

[5] Steinfeld, A. and Schubnell, M., "Optimum aperture size and operating temperature of a solar cavity-receiver," Solar Energy, 50(1), pp. 19-25, (1993).

[6] Petrasch, J., Osch, P., and Steinfeld, A., "Dynamics and control of solar thermochemical reactors," Chemical Engineering Journal, 145(3), pp. 362-370, (2009).

[7] Saade, E., Clough, D., and Weimer, A.W., "Model predictive control of a solar-thermal reactor," Solar Energy, 102, pp. 31-44, (2014).

[8] Li, L., Chen, C., Singh, A., Rahmatian, N., AuYeung, N., Randhir, K., Mei, R., Klausner, J.F., Hahn, D.W., and Petrasch, J., "A transient heat transfer model for high temperature solar thermochemical reactors," International Journal of Hydrogen Energy, 41, pp. 2307-2325, (2016).

[9] Klaij, C.M., "On the stabilization of finite volume methods with co-located variables for incompressible flow," Journal of Computational Physics, 297, pp. 84-89, (2015).

[10] Zhang, S., Zhao, X., Bayyuk, S., "Generalized formulations for the Rhie-Chow interpolation,” Journal of Computational Physics, 258, pp. 880-914, (2014).

[11] Zhang, J.K., Dong, H., Zhou, E.Z., Li, B.W., Tian, X.Y., "A combined method for solving 2D incompressible flow and heat transfer by spectral collocation method and artificial compressibility method," International Journal of Heat and Mass Transfer, 112, pp. 289-299, (2017).

[12] Patankar, S.V., Numerical Heat Transfer and Fluid Flow, New York: McGraw-Hill, pp. 30-135, (1980).

[13] Ophoff, C., Ozalp, N., "A novel iris mechanism for solar thermal receivers," Journal of Solar Energy Engineering, 139(6), pp. 1-10, (2017).

[14] Ballestrin, J., Ulmer, S., Morales, A., Barnes, A., Langley, L.W., and Rodriguez, M., "Systematic error in the measurement of very high solar irradiance," Solar Energy Materials and Solar Cells, 80(3), pp. 375-381, (2003).

[15] Ophoff, C., Korotunov, S. and Ozalp, N., "Optimization of Design and Process Parameters for Maximized and Stable Solar Receiver Efficiency," Proceedings of 2nd Thermal and Fluid Engineering Conference, TFEC-IWHT2017-18225, pp. 1-15, (2017).

[16] Mahan, J.R., Radiation Heat Transfer: A Statistical Approach, New York: John Wiley \& Sons, pp. 185-370, (2002).

[17] Modest, M.F., Radiative Heat Transfer, New York: Elsevier, pp. 836-846, (2013).

[18] Bird, R.B., Stewart, W.E., and Lightfoot, E.N., Transport Phenomena, New York: John Wiley \& Sons, pp. 20-140, (2006).

[19] American National Standards Institute (ANSI), American National Standard for Expressing Uncertainty - U.S. Guide to the Expression of Uncertainty in Measurement, ANSI/NCSL Z540-2-1997, (1997). 